\title{
Preliminary reference intervals and the impact of citrate storage time for thrombelastography in cats including delta and the velocity curve
}

\author{
Carolin Engelen ${ }^{1 *}$ (D), Andreas Moritz ${ }^{1}$, Franziska Barthel ${ }^{2}$ and Natali Bauer ${ }^{1}$
}

\begin{abstract}
Background: Thrombelastography is a useful tool in assessment of hemostasis. Beside the traditional variables, the velocity curve and the variable delta have lately earned attention. The velocity curve provides knowledge about the speed of clot formation including information about thrombin generation. Delta, which only reflects enzymatic coagulation, allows the determination of the origin of hypercoagulability when compared to clot rigidity, a variable that reflects both platelet and enzymatic activity. The aim was to establish preliminary reference intervals for feline thrombelastography including the velocity curve variables and delta obtained after 60 min of storage including the assessment of coefficients of variation. Furthermore, the effect of citrate storage time (30 versus 60 min) on feline thrombelastography will be determined.
\end{abstract}

Results: Prolonged storage times significantly reduced reaction $(R)(P=0.019)$ and clotting $(K)(P=0.008)$ times, split point $(\mathrm{SP})(P=0.019)$ and time to maximum rate of thrombus generation (TMRTG) $(P=0.023)$ values whereas maximum rate of thrombus generation (MRTG) significantly increased $(P=0.040$ ). Preliminary reference intervals: $R(\mathrm{~min}): 2.7-18.1 ; \mathrm{K}$ (min): 0. 8-3.9; alpha ( $\left(^{\circ}\right)$ : 27.6-75.2; maximum amplitude $(\mathrm{mm}): 18.5-62.5$; clot rigidity $\left(\mathrm{dyn} / \mathrm{cm}^{2}\right)$ : 1.2-8.2; coagulation index: -4.6 2.6; SP (min): 2.4-15.4; delta (min): 0.3-3.1; thrombus generation ( $\mathrm{mm} / \mathrm{min}): 255.3-751.2 ;$ MRTG (mm/min): 4.0-19.3; TMRTG (min): 3.5-22.0; maximum rate of lysis ( $\mathrm{mm} / \mathrm{min}): 0.0-4.7$ and time to maximum rate of lysis (min): 0.4-55.8.

Conclusion: Storage for 60 versus 30 min induces hypercoagulable tracings including the velocity curve, some of which variables (MRTG, TMRTG) might function as sensitive markers for changes in the coagulation activity. Because of the impact of citrate storage time on thrombelastography, reference intervals have to be established using a specific and constant storage time in each laboratory.

Keywords: Feline, Hypercoagulable, TEG, Velocity curve

\section{Background}

Thrombelastography (TEG) is a global coagulation test that enables assessment of hemostatic function in whole blood combining plasma and cellular components of hemostasis [1-3]. Recently, the global integrity of the complex process of blood coagulation is preferably assessed by measurement of thrombin generation [4], which can be evaluated using the TEG velocity curve (VC)

\footnotetext{
* Correspondence: Carolin.Engelen@vetmed.uni-giessen.de

'Department of Veterinary Clinical Sciences, Clinical Pathophysiology and

Clinical Pathology, Justus-Liebig University Giessen, Frankfurterstraße 126,

35392 Giessen, Germany

Full list of author information is available at the end of the article
}

[4] thus gaining new attention in human and veterinary medicine. The VC is generated from the mathematical first derivative of TEG values and dynamically reflects the velocity of clot formation (positive curve above the horizontal axis) and clot dissolution (negative curve below the horizontal axis) $[5,6]$ as illustrated in Fig. 1. Variables generated from the curve of clot formation include the maximum rate of thrombus generation (MRTG) represented by the peak of this curve [5]. In human medicine, MRTG has been shown to increase exponentially compared with the non-exponential and thus less pronounced increase of TEG alpha as a traditional marker of clot 


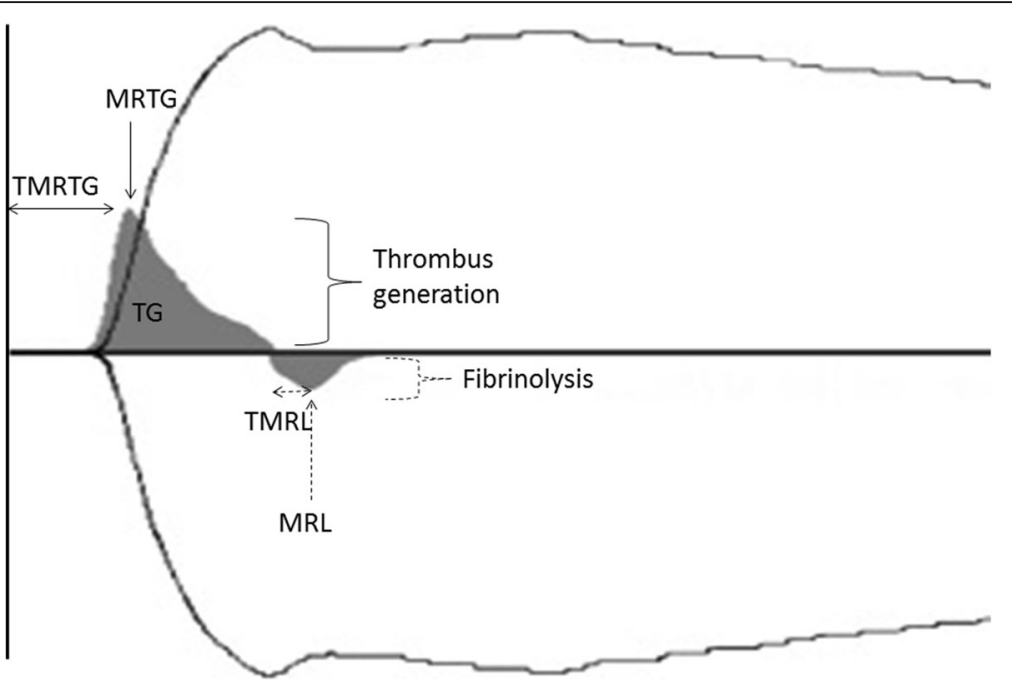

Fig. 1 Feline thrombelastography (TEG) showing the conventional tracing (black line) with superimposed velocity curve (gray solid curve). The gray curve above the horizontal axis reflects thrombus generation while the gray curve below represents fibrinolysis. Measurements that can be estimated include TMRTG (time to maximum rate of thrombus generation; min), MRTG (maximum rate of thrombus generation; $\mathrm{mm} / \mathrm{min}$ ), TG (thrombus generation; $\mathrm{mm} / \mathrm{min}$ ) from the area under the curve (AUC), TMRL (time to maximum rate of lysis; $\mathrm{mm} / \mathrm{min}$ ) and MRL (maximum rate of lysis; min)

formation, thus allowing a more precise evaluation of clot kinetics [7]. Other variables reflecting clot formation derived from the velocity curve include the time to maximum rate of thrombus generation (TMRTG), defined as the distance on the $\mathrm{x}$-axis from zero to the peak of the curve, and the thrombus generation (TG), which is calculated from the area under the curve and represents an indirect measurement of clot strength [5]. TMRTG is also believed to be a good marker of thrombin generation in humans and shows a linear correlation with the TEG parameter delta [6]. Delta is easily obtained from the TEG tracing by calculating the difference between two parameters R and SP of the traditional TEG curve [6] as shown in Fig. 2. Delta represents the greatest clot growth depending on peak thrombin generation, thus allowing the assessment of thrombin generation [6]. In people, the variable TG has been shown to correlate with thrombinantithrombin complex values which serve as a marker of thrombin generation [4]. Values that can be obtained from the curve below the horizontal axis reflect fibrinolysis and include the maximum rate of lysis (MRL) represented by the negative peak of this curve and the time to maximum rate of lysis (TMRL), which is the represented on the $\mathrm{x}$-axis from the start of the fibrinolysis curve to its negative peak [5].

The TEG software provides two modes of calculating the $\mathrm{VC}$ variables: first, $\mathrm{VC}$ variables can be calculated on the basis of the maximum amplitude (MA), a traditional TEG variable. Second, VC variables can be derived from the clot rigidity $(G)$, which itself is calculated from MA and represents the global coagulation activity. Like MA, G is dependent on factor XIII activity, concentration of fibrinogen and thrombin, the platelet count and function, and hematocrit [8].

Both versions (MA and G version) of calculation result in slightly different curves and subsequently different results. Furthermore, the variable names differ: $\mathrm{G}$ version variables have a "G" appended to the end of the name, i.e. MRTGG instead of MRTG [9].

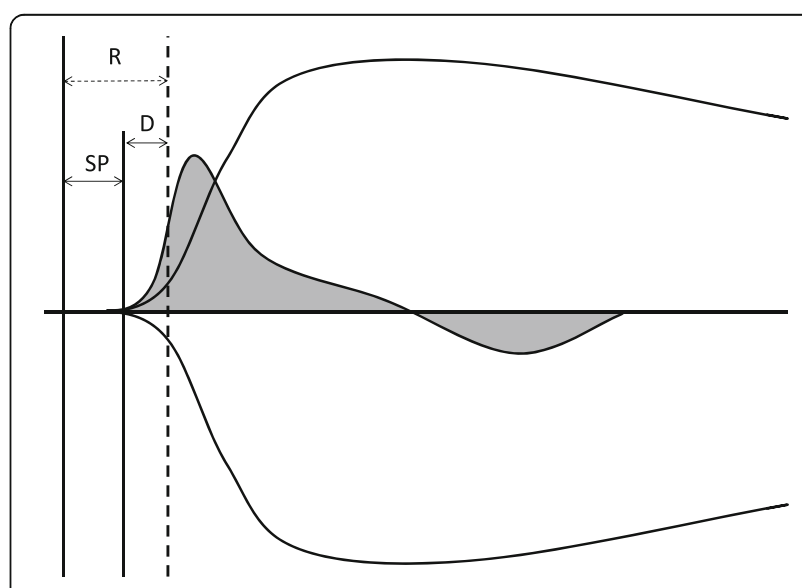

Fig. 2 Schematic representation of the variable delta (D; min; black arrow), which can be calculated from the difference between the reaction time $(R$; min; dotted arrow), i.e., the time from initiation of coagulation until the amplitude of the conventional TEG tracing reaches $2 \mathrm{~mm}$ and the split point (SP; min; longer black arrow), i.e., the time from starting the coagulation process until divergence of the TEG tracings from the zero line. The delta value $=\mathrm{R}-\mathrm{SP}$ 
From the clinical point of view, the TEG gained special interest for the evaluation of hypercoagulability [10], whereby it has an exceptional ability to detect individuals affected by or at risk for a thromboembolic event $[10,11]$. In human medicine, hypercoagulable states measured by rapid thrombelastography have already been shown to be a good predictor of thromboembolic events [12]. To help differentiating the contribution of enzymatic activity (reflecting secondary hemostasis) and platelet reactivity (reflecting primary hemostasis) in hypercoagulable states, the novel variable delta which represents the enzymatic activity has been introduced [6]. In correlation with the variable G (reflecting both primary and secondary hemostasis), hypercoagulable states can be classified as predominantly caused by platelet hypercoagulability if the patients show a hypercoagulable $\mathrm{G}$ value and a normal delta [6]. Neither delta nor the VC variables have yet been evaluated in cats.

For interpretation of TEG results, pre-analytical factors such as the sample material and the time between sampling and TEG analysis have to be taken into account. Originally designed to be run with fresh whole blood [3, 13], TEG analysis in veterinary medicine is mostly done using re-calcified citrated whole blood [10, 14]. Because citrate does not entirely prevent thrombin generation since the generation of factor XIIa (via contact activation) is not dependent on calcium, contact activation is not inhibited in citrated samples and continues during storage, resulting in a certain degree of thrombin generation [8, 13]. Consequently, the citrate storage time has an impact on TEG results [13], as demonstrated previously for people, dogs and horses [3, 15-18]. The impact of citrate storage time on feline TEG is largely unknown. Even if a storage time of $30 \mathrm{~min}$ has been recommended [13] and has mostly been used in previous investigations in cats [19-24], a storage time of $60 \mathrm{~min}$ might be preferable in some clinical settings especially if samples are used for a variety of different coagulation tests.

Thus, one aim of this study was to establish preliminary reference intervals (RIs) including assessment of coefficients of variation (CVs) for traditional and novel feline TEG parameters using $60 \mathrm{~min}$ incubation. The second aim was to determine the effect of citrate storage time (30 versus $60 \mathrm{~min}$ ) on TEG results which, to our knowledge, has not yet been done before in cats.

\section{Methods}

\section{Study design}

This prospective study was performed September 2015 and ethical approval has been given by the State Agency for Nature, Environment and Consumer Protection of North Rhine-Westphalia (LANUV NRW, reference number: 84-02.05.20.13.090, internal number: 200a176/232). Blood samples were collected from 21 clinically healthy adult domestic shorthair cats from Bayer Animal Health $\mathrm{GmbH}$ (Leverkusen, Germany) including 12 female and 9 male cats with a mean age of 12.3 months (range 12-13 months). The cats have been obtained from one breeder (Liberty Research, Waverly, US) and were kept under defined housing conditions in groups according to their gender. A commercially available food was fed (Josera Léger, Adult light, Kleinheubach, Germany) and water was available ad libitum.

Initial health status of the cats was determined through a routine physical examination. The blood was collected from the cephalic vein using a $21 \mathrm{G}$ needle $(0.8 \times 16.0 \mathrm{~mm}$, Neolus needle, Terumo, Eschborn, Germany) and dropped into two 1.4-ml 3.2\% sodium citrate tubes (S-Monovette ${ }^{\oplus}$, 1.4-ml, 9NC, Sarstedt, Wedel, Germany) which were properly filled ensuring an ratio of blood to anticoagulant of 9:1. In a randomized order, one tube of citrated whole blood was used for TEG analysis. From the other tube, citrated plasma for a routine coagulation profile including thrombin time (TT), prothrombin time (PT), activated partial thromboplastin time (aPTT), fibrinogen concentration and D-Dimers was obtained within $20 \mathrm{~min}$ after sampling by centrifugation (Eppendorf 5810 R, Eppendorf AG, Hamburg, Germany) for $10 \mathrm{~min}$. The plasma was then transferred into plain tubes (Eppendorf Safe-Lock Tubes, Eppendorf AG, Hamburg, Germany) and frozen at $-80{ }^{\circ} \mathrm{C}$. For coagulation analyses, frozen samples were shipped on dry ice to the department of Veterinary Clinical Sciences, Clinical Pathophysiology and Clinical Pathology (Justus-Liebig University, Giessen, Germany), and stored at $-80{ }^{\circ} \mathrm{C}$ until measurements were performed as batch on the automated coagulation analyzer STA Compact $^{\circledR}$ (Stago Germany, Düsseldorf, Germany) within a year after sample collection as described previously for dogs [25].

One week prior to the study, a complete blood count (CBC) measured with the automated hematology analyzer ADVIA $^{\star} 120$ Hematology System (Siemens HealthCare $\mathrm{GmbH}$, Erlangen, Germany; software version: no. 1) which has been evaluated previously for cats [26] and a biochemistry profile (IDEXX Catalyst $\mathrm{Dx}^{\oplus}$, IDEXX $\mathrm{GmbH}$, Ludwigsburg, Germany) were performed. Results were compared with RIs provided by the manufacturer of the clinical chemistry analyzer. Cats were included in our study when they were considered healthy based on the physical examination and the absence of clinically relevant abnormalities of the hematological and blood chemical examination.

The hematocrit and platelet count were reevaluated from citrated blood at the day of blood sampling for TEG analysis. Results were corrected by the factor 1.1 to take the dilution of the sample with citrate into consideration. The hematocrit value was evaluated with the microhematocrit method after centrifugation of $5 \mathrm{~min}$ at 
31,689 G using a microhematocrit-centrifuge ("Haematokrit 24", Hettich, Tuttlingen, Germany). The platelet count was estimated semiquantitatively by counting platelets in the monolayer of a May-Grünwald-Giemsa stained blood smear, whereby the mean of 10 high power fields (100× objective) was calculated and multiplied by a factor of 20 as previously recommended [27] to obtain the approximate platelet count $\left(\times 10^{3} / \mu \mathrm{l}\right)$. Thrombocytopenia was defined as values below 200 $\left(\times 10^{3} / \mu \mathrm{l}\right)$ [26]. When moderate to marked platelet clumping was seen, the assessed count was judged falsely low. Moderate to marked platelet clumping was defined as a high number of platelets within clumps that did not allow accurate counting of individual platelets [27]. As an addition to the size of clumps, the number of clumps was used to further classify the platelet clumping as low, moderate or high grade clumping. According to this classification, 13 cats were categorized as having low grade, 2 cats as moderate and 5 cats as having high grade platelet clumping.

\section{TEG analysis}

The analyses were always performed by the same person (CE) using three computerized TEG 5000 analyzers (Thrombelastograph, Haemonetics Corporation, Braintree, MA, USA). TEG analyses were done with recalcified whole blood stored at room temperature and according to the manufacturer recommendations. Briefly, standard TEG cups (Haemoscope Corporation) were placed in the $37^{\circ} \mathrm{C}$ prewarmed instrument holder and filled with $20 \mu \mathrm{l}$ of $0.2 \mathrm{M}$ calcium chloride. After specific storage times of either 30 and/or $60 \mathrm{~min}$ respectively, $340 \mu \mathrm{l}$ citrated whole blood was added and analysis was started. Each TEG analysis was performed for approximately $60 \mathrm{~min}$ and an electric internal quality control (e-test) was performed at least three times a day.

Blood from the first 10/21 cats was stored for $60 \mathrm{~min}$ before TEG analysis, which was run in duplicates using blood from the same tube to assess the variance. TEG assays for the remaining 11/21 cats were performed with blood taken from the same tubes after 30 and 60 min of storage time respectively to assess sample stability. One of these 11 cats had to be excluded from the study since TEG analysis only showed flat lines.

Evaluated TEG variables include the traditional variables: reaction time $(\mathrm{R})$, clotting time $(\mathrm{K})$, alpha, maximum amplitude (MA), clot rigidity $(\mathrm{G})$, coagulation index $(\mathrm{CI})$ and clot lysis at 30 min after MA is reached (LY30) as well as the novel measurements: SP, delta and the values from the velocity curve (TG, MRTG and TMRTG reflecting thrombin generation and MRL and TMRL reflecting clot lysis). The VC parameters used for all statistical analyses and figures were obtained from the MA version of calculation. For the sake of better comparison with other studies and potential future use by other investigators, reference intervals of $\mathrm{VC}$ parameters will be provided for both the $\mathrm{G}$ and MA version of calculation.

\section{Statistical analysis}

Preliminary RIs were established for a citrate storage time of $60 \mathrm{~min}$. If samples were measured in duplicates (as done for assessment of variance), the first result was used. Data distribution regarding normality was assessed by reviewing the histogram and the QQ plot as well as using the Anderson-Darling test or a symmetry test for Robust provided by the software (Reference Value Advisor 2.1 [28]). For all variables except for TG and TMRL, a robust method after Box-Cox transformation (Reference Value Advisor 2.1 [28]) was used. For TG, the RI was calculated with a parametric method after Box-Cox transformation and RI for TMRL was established by use of a parametric method of untransformed data respectively. Mean, median, minimum and maximum values as well as standard deviation was determined from untransformed data. Pooled variance was used to assess the percentage of total variation that occurred between the duplicates.

For all statistical comparisons, a Shapiro-Wilk test was done in advance to evaluate the assumption of normality. The impact of citrate storage times was assessed using a paired t-test for alpha, G, CI, TG, MRTG and TMRTG and a Wilcoxon test for R, K, MA, LY30, SP, delta, TMRL, MRL respectively. Because of the low number of cats in the group of high grade clumping the assessment of normality is limited so the influence of platelet clumps on the results was assessed by a nonparametric test (MannWhitney $\mathrm{U}$ test) for all variables. For all analyses, a $p$-value $<0.05$ was considered significant.

\section{Results}

\section{Hematological analyses}

The platelet count (PLT) estimated from the blood smear showed a thrombocytopenia $\left(36-124 \times 10^{3} / \mu \mathrm{l}\right)$ in five cats, four of which (PLT: $44-124 \times 10^{3} / \mu \mathrm{l}$ ) had marked aggregation on the blood smear so the count was considered falsely low. The remaining cat showing a thrombocytopenia $\left(36 \times 10^{3} / \mu \mathrm{l}\right)$ only had a moderate degree of platelet clumping. This cat did not show a thrombocytopenia on the CBC 1 week earlier and was clinically healthy without a history of bleeding so it was still included in the study.

Re-evaluation of the corrected hematocrit on day of sampling demonstrated no major abnormalities.

\section{Analyses of plasmatic hemostasis}

Mean, median, range and standard deviation for TT, PT, aPTT, fibrinogen and D-dimers are shown in Table 1. 
Table 1 Results of the traditional coagulation profile obtained from 21 cats

\begin{tabular}{llllll}
\hline Variable & $T$ (sec) & PT $(\mathrm{sec})$ & aPTT $(\mathrm{sec})$ & Fibrinogen $(\mathrm{g} / \mathrm{l})$ & $\mathrm{D}$-dimers $(\mu \mathrm{g} / \mathrm{ml})$ \\
\hline Mean & 15.37 & 10.8 & 11.92 & 1.46 & 0.18 \\
Median & 15.4 & 10.8 & 12 & 1.48 & 0.2 \\
Range & $14-16.9$ & $10.1-12.8$ & $10.8-13$ & $1.04-1.7$ & $0.09-0.26$ \\
SD & 0.82 & 0.56 & 0.74 & 0.17 & 0.05 \\
RI (laboratory-internal ${ }^{\mathrm{a}}$ ) & $13.4-19.1$ & $9.9-12.2$ & $10.8-14.0$ & $0.6-2.2$ & $0.0-0.3$ \\
\hline Abbratin
\end{tabular}

Abbreviations: $S D$ standard deviation, $R I$ reference interval, $T$ thrombin time, $P T$ prothrombin time, aPTT activated partial thromboplastin time, sec seconds ${ }^{a}$ For interpretation of the values, laboratory-internal reference intervals (RIs) established from 40 clinically healthy domestic shorthair cats using a robust method of untransformed data are additionally listed

Compared to the laboratory internal RIs which are also listed in Table 1, all values were considered normal denoting a physiological secondary hemostasis in all cats.

\section{Preliminary Rls}

One cat $(1 / 21)$ had to be excluded because no TEG tracing could be obtained. This cat showed a marked aggregation of platelets on the blood smear and an estimated PLT count of $44 \times 10^{3} / \mu \mathrm{l}$. The routine coagulation parameters were unremarkable in this cat.

Preliminary RIs, CVs as well as the mean, median, the standard deviation, minimum and maximum for the investigated TEG variables of citrated whole blood samples stored for $60 \mathrm{~min}$ are shown in Table 2.

Histograms of the distribution of all evaluated variables after Box Cox transformation (LY30) or untransformed data (all but LY30) are shown in Figs. 3 and 4. In one cat $(1 / 20)$, the CI and K value were not reported by the TEG software and in another cat $(1 / 20)$ no results for MRL and TMRL were obtained. For LY30, data distribution did not allow the calculation of a reliable RI.

The CV was $<20 \%$ for the variables $R, K$, alpha, MA, G, SP, TG and MRTG. For the other evaluated variables
(CI, LY30, delta, TMRTG, MRL and TMRL) CVs were ranging between $23.6 \%$ (delta) and $83.6 \%(\mathrm{CI})$ respectively. Statistical analysis did not reveal a significant difference between the first and the second measurement performed after a citrate storage time of $60 \mathrm{~min}$ for all assessed variables.

\section{Impact of citrate storage time}

As seen in Figs. 5 and 6, citrate storage time had a significant impact on many traditional and novel TEG variables. When evaluating the traditional TEG variables (Fig. 5), prolonged citrate storage time (60 versus $30 \mathrm{~min}$ ) induced a significant decrease in median values of R ( $7 \mathrm{~min}$. vs. $12.8 \mathrm{~min} ., P=0.019)$ and $\mathrm{K}(2.5 \mathrm{~min}$. vs. 3.4 min., $P=0.008$ ) respectively. Interestingly, a marked increase in inter-individual variation of LY30 was noted with increasing citrate storage time. Regarding the novel variables (Fig. 6), the TMRTG was significantly shorter (8.92 min. vs. $15.17 \mathrm{~min} ., P=0.023$ ), the MRTG was significantly larger $(8.03 \mathrm{~mm} / \mathrm{min}$ vs. $6.26 \mathrm{~mm} / \mathrm{min}, P=$ $0.04)$ and the SP was significantly smaller $(5.9 \mathrm{~min}$. vs. 11.4 min., $\mathrm{P}=0.019)$ after $60 \mathrm{~min}$ when compared to results after $30 \mathrm{~min}$ of storage. Overall, these results reflect

Table 2 Shows preliminary reference intervals (RI) for all investigated trombelastography (TEG) variables

\begin{tabular}{|c|c|c|c|c|c|c|c|c|c|c|c|c|c|c|}
\hline $\begin{array}{l}\text { Variable } \\
\text { (unit) }\end{array}$ & $\begin{array}{l}R \\
(\min )\end{array}$ & $\begin{array}{l}\mathrm{K} \\
(\min )\end{array}$ & $\begin{array}{l}\text { alpha } \\
\text { (degree) }\end{array}$ & $\begin{array}{l}\text { MA } \\
(\mathrm{mm})\end{array}$ & $\begin{array}{l}\text { G (dyn/ } \\
\left.\mathrm{cm}^{2}\right)\end{array}$ & $\begin{array}{l}\mathrm{Cl} \\
\text { (unitless) }\end{array}$ & $\begin{array}{l}\text { LY30 } \\
(\%)\end{array}$ & $\begin{array}{l}\mathrm{SP} \\
(\min )\end{array}$ & $\begin{array}{l}\text { delta } \\
\text { (min) }\end{array}$ & $\begin{array}{l}\mathrm{TG}^{\mathrm{a}}(\mathrm{mm} / \\
\mathrm{min})\end{array}$ & $\begin{array}{l}\text { MRTG }^{\mathrm{a}}(\mathrm{mm} / \\
\mathrm{min})\end{array}$ & $\begin{array}{l}\text { TMRTG }^{a} \\
\text { (min) }\end{array}$ & $\begin{array}{l}\mathrm{MRL}^{\mathrm{a}}(\mathrm{mm} / \\
\min )\end{array}$ & $\begin{array}{l}\mathrm{TMRL}^{\mathrm{a}} \\
\text { (min) }\end{array}$ \\
\hline $\begin{array}{l}\text { Number of } \\
\text { cats }(n)\end{array}$ & 20 & 19 & 20 & 20 & 20 & 19 & 20 & 20 & 20 & 20 & 20 & 20 & 19 & 19 \\
\hline $\mathrm{Rl}$ & $\begin{array}{l}2.7- \\
18.1\end{array}$ & $\begin{array}{l}b \\
0.8- \\
3.9\end{array}$ & $\begin{array}{l}27.6- \\
75.2\end{array}$ & $\begin{array}{l}18.5- \\
62.5\end{array}$ & $1.2-8.2$ & $\begin{array}{l}b \\
-4.6- \\
2.6\end{array}$ & c & $\begin{array}{l}2.4- \\
15.4\end{array}$ & $\begin{array}{l}0.3- \\
3.1\end{array}$ & $\begin{array}{l}255.3- \\
751.2\end{array}$ & $4.0-19.3$ & $\begin{array}{l}3.5- \\
22.0\end{array}$ & $0.0-4.7$ & $\begin{array}{l}0.4- \\
55.8\end{array}$ \\
\hline Minimum & 2.8 & 1 & 26.1 & 18.2 & 1.1 & -4.6 & 2.4 & 2.4 & 0.3 & 244.87 & 3.48 & 3.67 & 0.13 & 9.58 \\
\hline Maximum & 18.7 & 3.6 & 74.9 & 61.4 & 8 & 2.5 & 15.8 & 15.8 & 2.9 & 735.09 & 19.28 & 22.5 & 4.37 & 56.08 \\
\hline Mean & 7.6 & 2.3 & 57.6 & 46.3 & 4.6 & -0.2 & 11.1 & 6.6 & 1.0 & 563.1 & 9.4 & 9.8 & 1.8 & 28.1 \\
\hline Median & 6.6 & 2.3 & 58.1 & 47.3 & 4.5 & 0.1 & 6.6 & 5.8 & 0.9 & 588.5 & 8.5 & 8.8 & 1.8 & 22.4 \\
\hline SD & 3.8 & 0.7 & 10.7 & 10.0 & 1.7 & 1.6 & 11.6 & 3.2 & 0.6 & 112.9 & 3.5 & 4.5 & 1.1 & 12.8 \\
\hline CV(\%) & 15.2 & 4.7 & 13.2 & 8.4 & 19.5 & 83.6 & 57.2 & 15.3 & 23.6 & 8.9 & 11.5 & 27.1 & 33.9 & 35.7 \\
\hline
\end{tabular}

Abbreviations: $R I$ reference interval, min minutes, $S D$ standard deviation, $C V$ coefficient of variation, $R$ reaction time, $K$ clotting time, $M A$ maximum amplitude, $G$ clot rigidity, $\mathrm{Cl}$ coagulation index, $L Y 30$ clot lysis at $30 \mathrm{~min}, S P$ split point, $T G$ total thrombin generation, MRTG maximum rate of thrombus generation, $T M R T G$ time to maximum rate of thrombus generation, $M R L$ maximum rate of lysis, TMRL time to maximum rate of lysis

${ }^{a}$ Velocity curve variables (TG, MRTG, TMRTG, MRL, TMRL) were obtained as MA version

${ }^{\mathrm{b}}$ Reference intervals are not considered valid according to the ASVCP (American Society of Veterinary Clinical Pathology) guidelines as number of animals $<20$

${ }^{\mathrm{C}} \mathrm{A}$ valid reference interval could not be calculated with any statistical method 
the development of a more hypercoagulable TEG tracing with increasing citrate storage time. For the variables of clot lysis, only MRL was significantly influenced by citrate storage time with values being higher at $60 \mathrm{~min}$ compared to $30 \mathrm{~min}(1.71 \mathrm{~mm} / \mathrm{min}$ vs. $1.25 \mathrm{~mm} / \mathrm{min}, P$ $=0.049)$. In contrast, there was no significant impact of citrate storage time on the other investigated TEG variables (alpha, MA, G, CI, delta, LY30, TG, TMRL).

Examples of two TEG tracings performed in a cat after a citrate storage time of 30 and $60 \mathrm{~min}$ respectively is depicted in Fig. 7 clearly demonstrating the decrease in $\mathrm{R}, \mathrm{K}, \mathrm{SP}$ and TMRTG as well as an increase in MRTG.

\section{Impact of platelet clumping}

No statistically significant differences could be found between the group of cats classified as having low grade clumping and those cats classified as showing high grade platelet clumping. Table 3 provides the range and median of the different groups as well as the $p$-value between these two groups.

\section{Discussion}

To the authors' knowledge, this is the first study evaluating novel TEG variables such as SP, delta and the VC variables for cats as well as the impact of storage time on feline TEG tracings.

There is no definite evidence why one cat (excluded from the study) only showed flat lines drawing the conclusion that no clot could be detected by the analyzer. Because the routine plasmatic coagulation parameters were unremarkable in this cat, a coagulation disorder seems unlikely. Relating to the TEG being a test of global hemostasis, a platelet origin might be the reason of undetectable clots in this cat. In fact, this cat showed marked aggregation of platelets on the blood smear, but since five different cats also showed moderate to marked platelet aggregation on the blood smear and TEG tracings could still be obtained, an aggregation of platelet might not be the only reason. Further studies should be obtained to verify this observation.

Our study demonstrated a significant impact of citrate storage time on several traditional and novel TEG variables. Consequently, RI's are also influenced by the citrate storage time and thus should be established for any citrate storage time intended to be used in the laboratory. However, when using the RI, the relatively high CVs of many TEG variables have to be taken into account.

One limitation of our study is the small number of cats included so that potential significant effects might have been missed. Moreover, the establishment of RIs is hampered by the small sample size and RIs determined here have to be considered as preliminary values. As recommended by the American Society of Veterinary Clinical Pathology (ASVCP) guidelines for reference samples $20 \leq x<40$, a robust method was mostly used for the establishment [29]. Furthermore, these guidelines suggest that reference intervals should not be obtained when less than 20 samples are available [29] as was the case for K, CI, MRL and TMRL. For reference samples $10 \leq x<20$, the ASVCP guidelines recommend to report histograms (Figs. 3 and 4) as well as

Table 3 Comparison of the TEG results of different groups of cats categorized by the grade of platelet clumping

\begin{tabular}{|c|c|c|c|c|c|c|c|}
\hline \multirow{2}{*}{$\begin{array}{l}\text { Group of cats } \\
\text { Variable (unit) }\end{array}$} & \multicolumn{3}{|c|}{ Low grade of clumping } & \multicolumn{3}{|c|}{ High grade of clumping } & \multirow{2}{*}{$\begin{array}{l}p^{-} \\
\text {value }\end{array}$} \\
\hline & Number of cats & Range & Median & Number of cats & Range & Median & \\
\hline $\mathrm{R}(\mathrm{sec})$ & 13 & $3.3-18.7$ & 6.5 & 5 & $5.8-9.6$ & 8.1 & 0.29 \\
\hline K (sec) & 12 & $1.4-3.4$ & 2.4 & 5 & $1.7-3.4$ & 2.3 & 0.67 \\
\hline Alpha $\left({ }^{\circ}\right)$ & 13 & $26.1-70$ & 57.8 & 5 & $49.3-66.4$ & 58.3 & 0.94 \\
\hline $\mathrm{MA}(\mathrm{mm})$ & 13 & $18.2-61.4$ & 46.6 & 5 & $42.1-60.9$ & 52.6 & 0.12 \\
\hline $\mathrm{G}\left(\mathrm{dyn} / \mathrm{cm}^{2}\right)$ & 13 & $1.1-8$ & 4.4 & 5 & $3.6-7.8$ & 5.5 & 0.11 \\
\hline LY30 (\%) & 13 & $0-36.8$ & 5.3 & 5 & $1.2-27.6$ & 7.9 & 0.92 \\
\hline $\mathrm{Cl}$ (unitless) & 12 & $-2.3-2.5$ & -0.2 & 5 & $-1.5-2.1$ & 0.3 & 0.34 \\
\hline $\mathrm{SP}(\mathrm{min})$ & 13 & $3-15.8$ & 5.7 & 5 & $5.2-8.6$ & 7 & 0.25 \\
\hline Delta (min) & 13 & $0.3-2.9$ & 0.8 & 5 & $0.6-1.1$ & 1 & 0.52 \\
\hline TMRTG (min) & 13 & $4.7-22.5$ & 8.8 & 5 & $7.1-11.9$ & 10.3 & 0.62 \\
\hline MRTG (mm/min) & 13 & $3.5-14.7$ & 8.1 & 5 & $6.2-12.2$ & 9.6 & 0.63 \\
\hline $\mathrm{TG}(\mathrm{mm} / \mathrm{min})$ & 13 & $244.9-735.1$ & 565 & 5 & $517.5-734.3$ & 636.3 & 0.12 \\
\hline TMRL (min) & 12 & $15.7-56.1$ & 21.2 & 5 & $22.3-46.3$ & 34.1 & 0.08 \\
\hline $\mathrm{MRL}(\mathrm{mm} / \mathrm{min})$ & 12 & $0.1-4.4$ & 1.8 & 5 & $0.3-2.5$ & 1.4 & 0.59 \\
\hline
\end{tabular}

Abbreviations: $S D$ standard deviation, $R$ reaction time, $K$ clotting time, $M A$ maximum amplitude, $G$ clot rigidity, $C l$ coagulation index, $L Y 30$ clot lysis at 30 min, $S P$ split point, $T G$ total thrombin generation, MRTG maximum rate of thrombus generation, TMRTG time to maximum rate of thrombus generation, MRL maximum rate of lysis, $T M R L$ time to maximum rate of lysis 

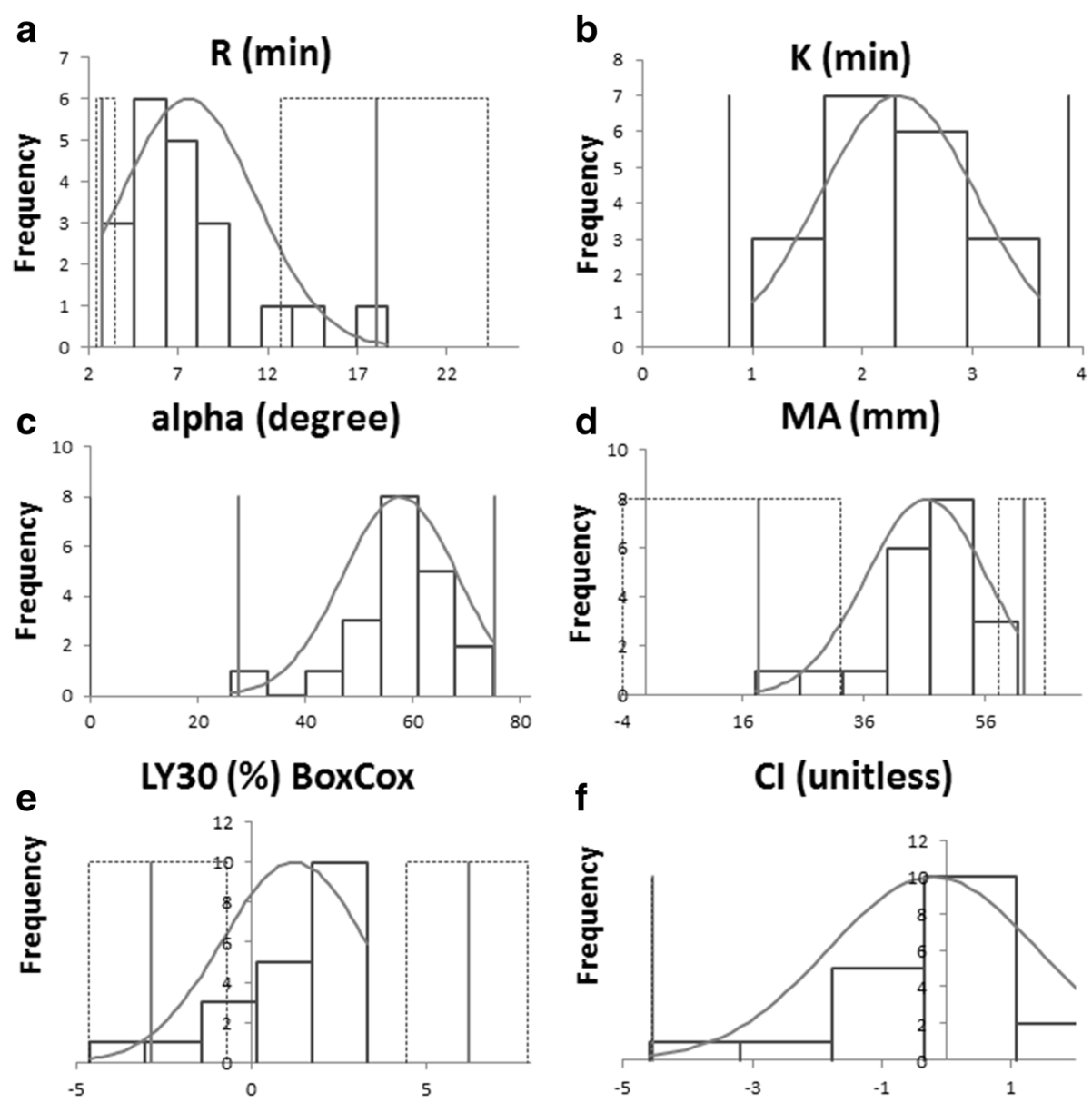

f
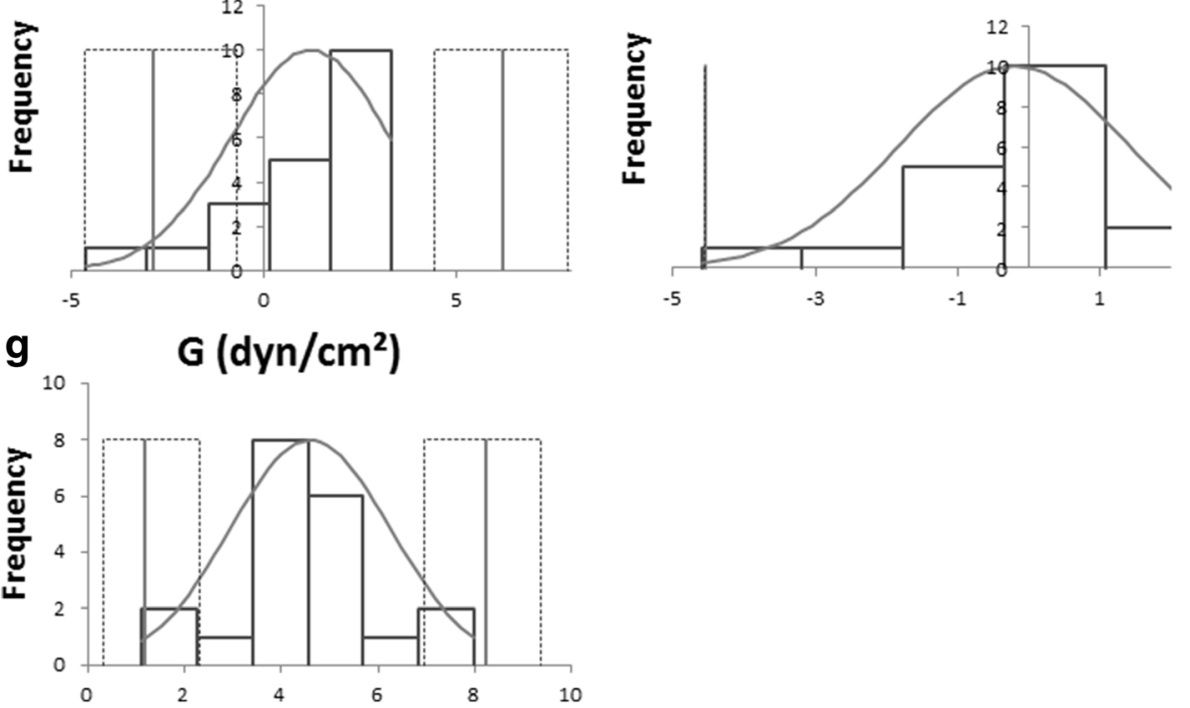

Fig. 3 Histograms showing distribution of untransformed (all but LY30) data obtained for calculation of the reference interval of the traditional TEG. Data for measurement included the first results of all samples after $60 \mathrm{~min}$ of storage time. The solid grey line represents the upper and the lower limit of the reference interval. The small black dotted line is consistent with the $90 \%$ confidence interval. The light grey line indicates the Gaussian curve. Abbreviations: $\mathrm{R}=$ reaction time $(\mathbf{a}), \mathrm{K}=$ clotting time $(\mathbf{b})$, Alpha $=$ alpha angle $(\mathbf{c}), \mathrm{MA}=$ maximum amplitude $(\mathbf{d}), \mathrm{LY} 30=$ lysis at $30 \min (\mathbf{e}), \mathrm{Cl}=$ coagulation index $(\mathbf{f}), \mathrm{G}=$ clot rigidity $(\mathbf{g})$

mean and median values (Table 2) to help facilitate interpretation of the values.

A direct comparison of RIs obtained in this study using a storage time of $60 \mathrm{~min}$ to the results of other studies cannot be made without serious limitations since other studies used a different citrate storage time (i.e., $30 \mathrm{~min}$ ) $[19,21]$ or different modes of activation $[19,21]$ which also can affect TEG variables. With regard to the velocity variables, no other study evaluating the $\mathrm{VC}$ in feline thrombelastography was found. For better comparability, Table 4 provides the RIs of both the MA version and G version of the velocity curve variables, which are slightly different since the curves (MA version and G version) are minimally different.

Regarding the stability of citrated whole blood, our study shows that longer storage times induce a more hypercoagulable TEG tracing which is not only represented by a shorter R, K and SP but also can be 

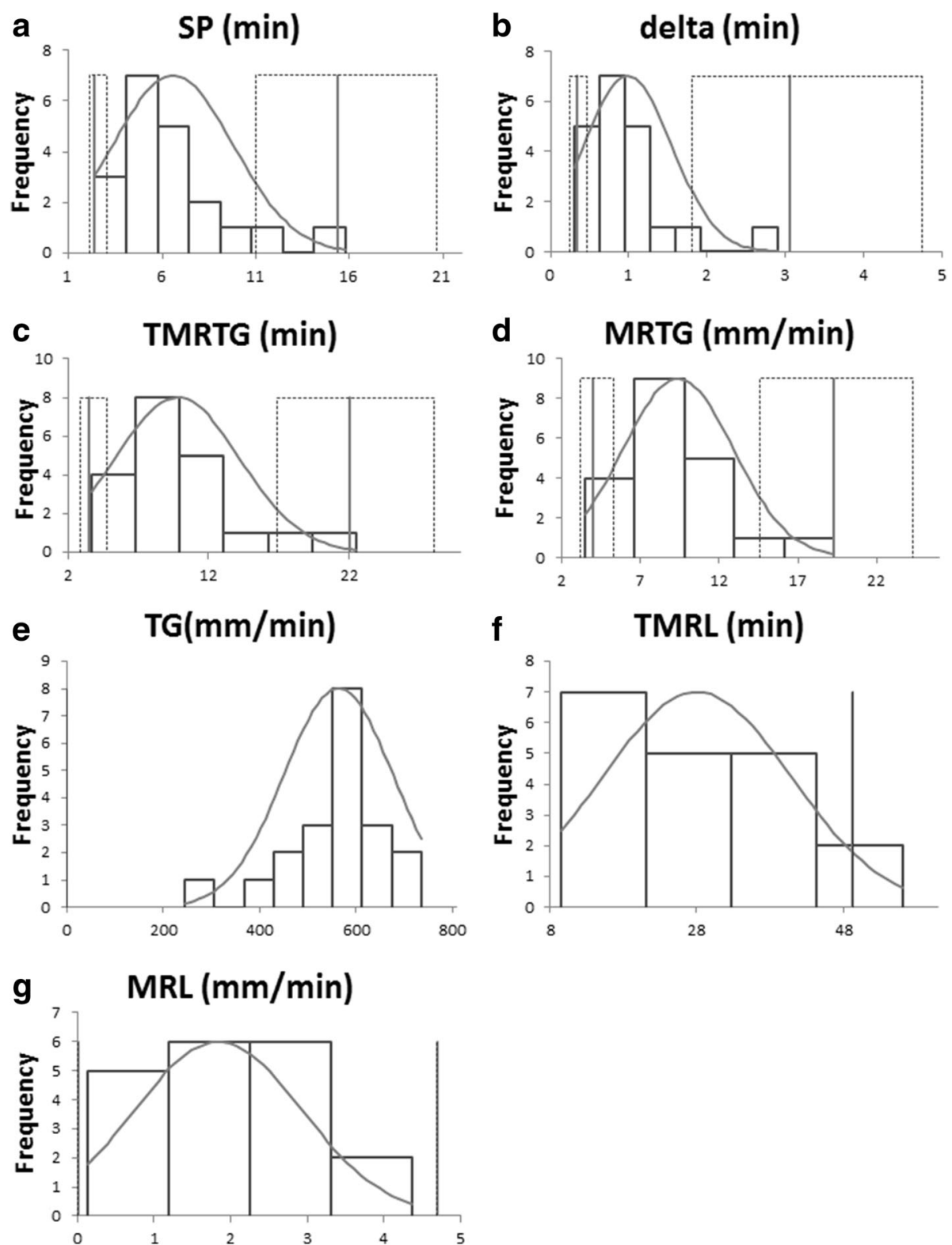

Fig. 4 Histograms showing the distribution of untransformed data for calculation of reference intervals of the novel TEG variables SP, delta, TMRTG, MRTG, TMRL and MRL respectively. Data for measurement included the first results of all samples after 60 min of storage time. The solid grey line represents the upper and the lower limit of the reference interval. The small black dotted line is consistent with the $90 \%$ confidence interval. The light grey line indicates the Gaussian curve. Abbreviations: SP = split point (a), D = delta (b), TMRTG = time to maximum rate of thrombus generation $(\mathbf{c}), M R T G=$ maximum rate of thrombus generation $(\mathbf{d}), T G=$ thrombus generation $(\mathbf{e})$, TMRL = time to maximum rate of lysis (f), MRL = maximum rate of lysis $(\mathbf{g})$

seen on the velocity curve by reduced TMRTG and MRTG values.

The $R$ value reflects the time from sample placement in the cuvette until an amplitude of $2 \mathrm{~mm}$ is reached on the TEG tracing. It is dependent on plasma clotting factors and circulating inhibitor activity and represents the initial fibrin formation rate [1]. The $K$ value is a measurement from the point where $2 \mathrm{~mm}$ of amplitude is reached ( $\mathrm{R}$ time) to the point of $20 \mathrm{~mm}$ amplitude.
This time reflects the buildup and cross linking of fibrin, which is related to clotting factors such as factor II and VII, fibrinogen and platelets count and function [1].

Furthermore, alpha and delta show a non-significant trend towards hypercoagulability at $60 \mathrm{~min}$. Alpha $\left(^{\circ}\right)$ is the angle formed by the TEG tracing between the R and the $K$ value and reflects the speed of clot formation [1]. As alpha and MRTG reflect a similar item, a similarly significant change could have been expected between 
a

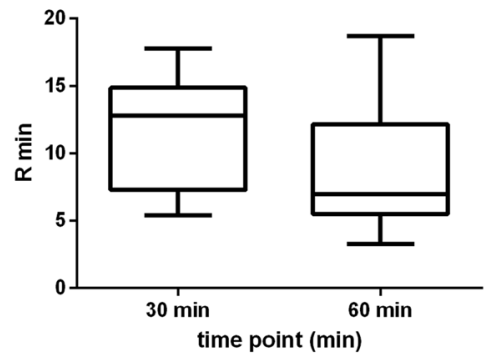

C

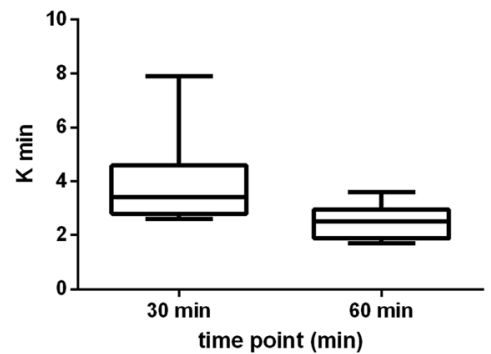

e

$P=0.92$

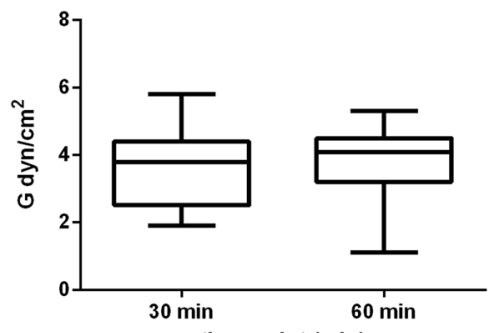

time point (min) b $\quad P=0.086$

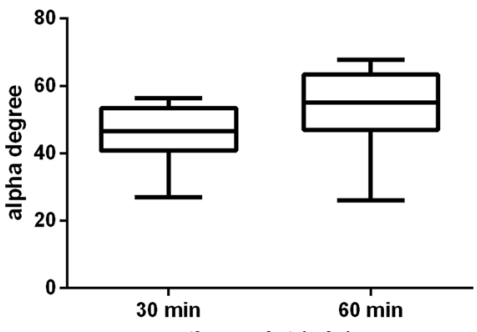

time point (min)

d

$\mathrm{P}=0.83$

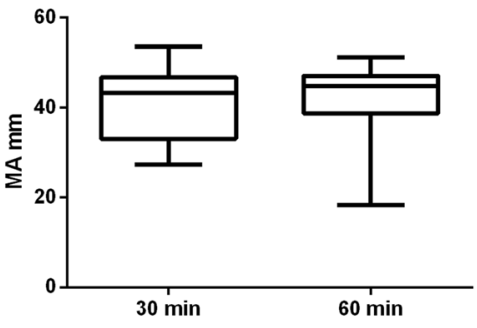

time point $(\min )$

f

$P=0.97$

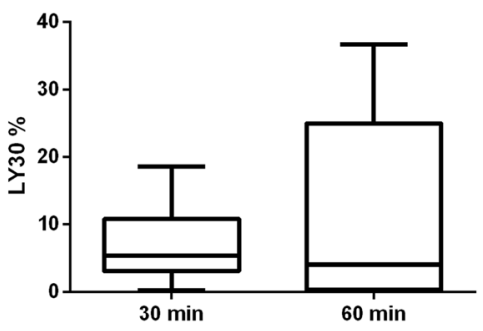

time point ( $\mathrm{min}$ )
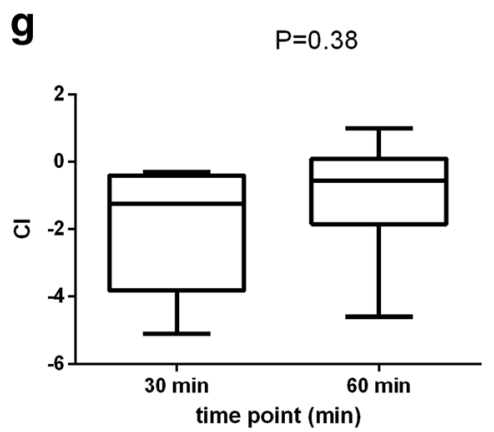

Fig. 5 Effect of citrate storage time (30 min versus $60 \mathrm{~min}$ ) on the traditional thrombelastographic (TEG) variables expressed as box and whisker diagrams. The central box represents values from the lower (25th) to upper (75th) percentile, while the middle line is consistent with the median. The horizontal line depicts the minimum and maximum values. The level of significance was set at $P<0.05$. Abbreviations: $R=$ reaction time (a), alpha degree $(\mathbf{b}), \mathrm{K}=$ coagulation time $(\mathbf{c}), \mathrm{MA}=$ maximum amplitude $(\mathbf{d}), \mathrm{G}=$ clot rigidity $(\mathbf{e}), \mathrm{LY} 30=$ lysis at $30 \mathrm{~min}(\mathbf{f}), \mathrm{Cl}=$ coagulation index $(\mathbf{g})$

the time points. However, alpha was not significantly altered while MRTG was significantly affected by different storage times. A possible explanation for this discrepancy could be their different mode of change during the coagulation process as described previously for humans [7]: compared to alpha which is variable of the traditional curve and shows a non-exponential behavior, MRTG as a parameter derived from the 
a

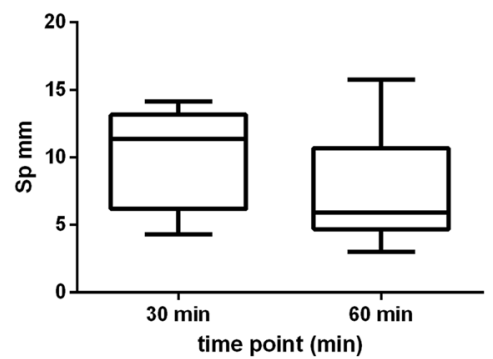

C

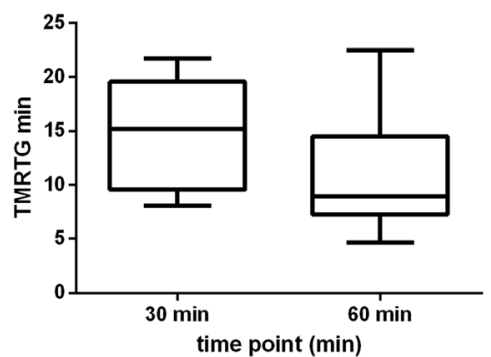

e

$\mathrm{P}=0.72$

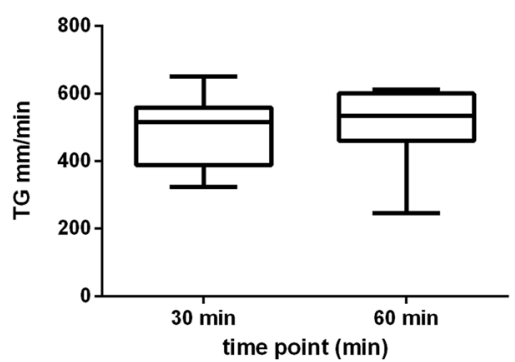

b

$\mathrm{P}=0.088$

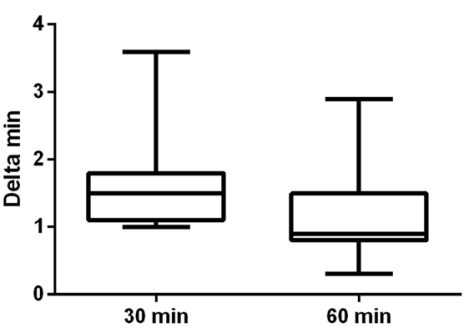

time point ( $\mathrm{min})$
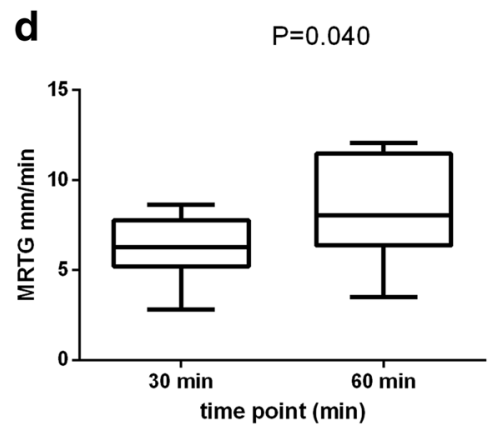

f

$P=0.49$

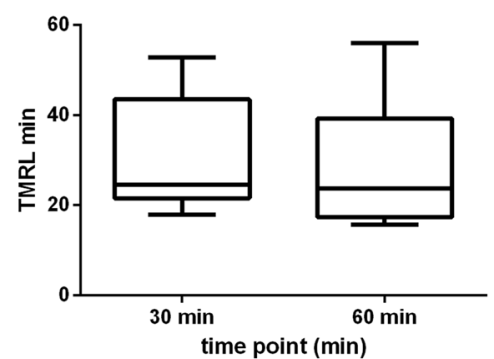

g $\quad \mathrm{P}=0.049$

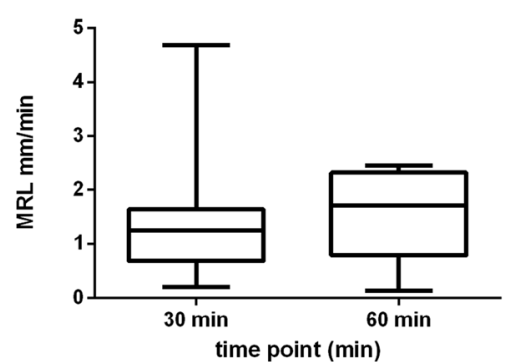

Fig. 6 Effect of citrate storage time (30 min versus $60 \mathrm{~min}$ ) on the novel thrombelastographic (TEG) variables expressed as box and whisker diagrams. The central box represents values from the lower (25th) to upper (75th) percentile, while the middle line is consistent with the median. The horizontal line depicts the minimum and maximum values. The level of significance was set at $P<0.05$. Abbreviations: SP=split point (a), D = delta (b), TMRTG = time to maximum rate of thrombus generation (c), MRTG = maximum rate of thrombus generation $(\mathbf{d}), \mathrm{TG}=$ thrombus generation $(\mathbf{e}), T M R L=$ time to maximum rate of lysis $(\mathbf{f}), M R L=$ maximum rate of lysis $(\mathbf{g})$ 


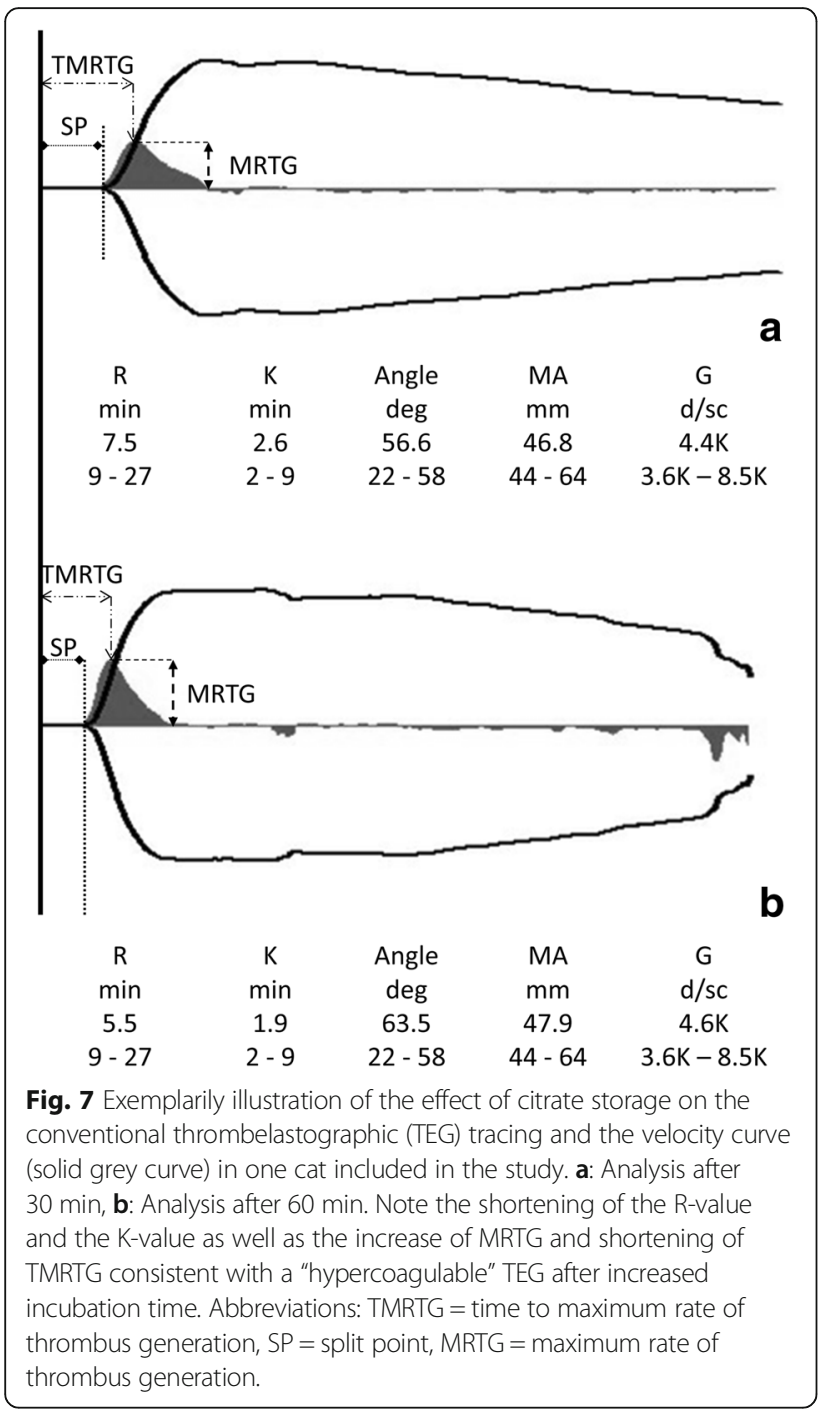

velocity curve changes in an exponential and thus markedly pronounced way.

A similar effect was noticed here for TMRTG which in previous studies in human medicine has shown a linear correlation with delta [6] so that theoretically a similar impact of storage on both variables would have been expected. However, the citrate storage time only had a significant impact on the VC parameter TMRTG increasing exponentially but not on delta derived from the traditional TEG curve changing in a non-exponential mode. In conclusion, some VC variables (TMRTG, MRTG) might be a more sensitive marker of changes in coagulation activity like hypercoagulability than traditional TEG values. This hypothesis however, further has to be substantiated in future clinical studies.

A previous investigation in critically ill patients could further demonstrate a linear relationship between delta and TMRTG reflecting thrombin generation [6]. In our study evaluating the impact of citrate storage time in
Table 4 Comparison of the velocity curve reference intervals based on the maximum amplitude or clot rigidity

\begin{tabular}{llll}
\hline MA version & \multicolumn{3}{l}{ G version } \\
\hline Variable (unit) & $\mathrm{Rl}$ & Variable (unit) & $\mathrm{Rl}$ \\
TMRTG (min) & $3.5-22.0$ & TMRTGG (min) & $4.1-23.0$ \\
MRTG (mm/min) & $4.0-19.3$ & MRTGG (dsc) & $2.1-11.9$ \\
TG (mm/min) & $255.3-751.2$ & TGG (dsc) & $138.8-828.5$ \\
TMRL (min) & $0.4-55.8$ & TMRLG (min) & $0.0-55.9$ \\
MRL (mm/min) & $0.0-4.7$ & MRLG (dsc) & $-1.6-7.7$ \\
\hline
\end{tabular}

For each variable, in both versions the same statistical method was used. G version variables show an appended " $\mathrm{G}$ "

Abbreviations: $M A$ maximum amplitude, $G$ clot rigidity, $R /$ reference interval, TMRTG(G) time to maximum rate of thrombus generation, $M R T G(G)$ maximum rate of thrombus generation, $T G(G)$ (total) thrombus generation, $T M R L(G)$ time to maximum rate of lysis, $M R L(G)$ maximum rate of lysis, $\min$ minute, $\mathrm{mm}$ millimeter, dsc dyn $/ \mathrm{cm}^{2} / \mathrm{s}$

TEG variables, the behavior of TMRTG resembled the findings observed for $\mathrm{R}$ more closely than those observed for delta. It thus could be hypothesized, that there is an even closer relationship between TMRTG and $\mathrm{R}$ than there is between TMRTG and delta. Reasons contributing to this difference in the current study compared to Gonzalez et al. [6] might be the different species, the different storage time, the different mode of activation and the prophylactic therapy with heparin potentially influencing the values as $\mathrm{R}$ partially depends on the activity of circulating inhibitors [1]. Further studies are needed to evaluate the relationship amongst this threesome (TMRTG, $\mathrm{R}$ and delta).

Regarding TG, previously investigated in humans as a marker of thrombin generation [4], the absolute amount of thrombus generated (TG) in our study in cats was interestingly not altered by prolonged citrate storage times. In conjunction with the changes in TMRTG and MRTG, citrate storage time apparently rather affects the speed of clot formation and thrombin generation (as TMRTG is believed to be a marker of thrombin generation [6]) than the total amount of thrombin generated.

In agreement with our findings in cats, significant differences with a tendency towards hypercoagulability with longer storage time have been observed in dogs [3] and horses [17]. In contrast to that, another study in horses reported sample (citrated whole blood) stability at room temperature for up to $20 \mathrm{~h}$ prior to TEG measurement, however, measurements were made after 2 and 19 to $48 \mathrm{~h}$ of citrate storage time so that changes prior $2 \mathrm{~h}$ of storage might have been missed [18].

Despite from an incomplete inhibition of thrombin formation during storage [3], repeated sampling from a single tube could function as a "contact activator" for the coagulation cascade via the pipette surface which in humans has been shown to alter TEG 
results [16]. The factor of repeated sampling has not been determined in our study but in other veterinary studies which used separate tubes for different time points to avoid repeated pipetting and still showed significant differences with a tendency towards hypercoagulability [3, 17]. Hypercoagulable TEG tracings with prolonged storage times might thus be resulting from different in vitro blood stability of different mammalian species [17].

\section{Conclusion}

Storage of citrated whole blood used for TEG analysis in cats has significant effects on both traditional and VC variables with a trend towards hypercoagulability with prolonged storage times. By that, some velocity curve variables (explicitly TMRTG, MRTG) might help in the early detection of changes in coagulation activity for example hypercoagulable states.

Because of the effect of citrate storage times on TEG analysis, RIs can only be used for a specific storage time which has to be established in each laboratory.

\section{Abbreviations}

aPTT: activated partial thromboplastin time; Cl: coagulation index $\mathrm{cm}$ : centimeter; CV: coefficients o variation; dsc: dyn $/ \mathrm{cm}^{2} / \mathrm{s}$; G: clot rigidity; $\mathrm{K}$ : clotting time; LY30: lysis at 30 min after MA is reached; MA: maximum amplitude; min: minute; $\mathrm{mm}$ : millimeter; MRL: maximum rate of lysis; MRTG: maximum rate of thrombus generation; PT: prothrombin time; R: reaction time; RIs: reference intervals; sec: second; SP: split point; TEG: thrombelastography; TG: (total) thrombus generation; TMRL: time to maximum rate of lysis; TMRTG: time to maximum rate of thrombus generation; T: thrombin time; $\mathrm{VC}$ : velocity curve

\section{Acknowledgements}

The authors would like to thank Bayer Animal Health GmbH, Leverkusen for kindly supporting this study.

\section{Funding}

The study was kindly supported by Bayer Animal Health GmbH by providing the equipment and material necessary for blood sampling and plasma preparation. No author received financial support for the authorship or publication of this article.

\section{Availability of data and materials}

The datasets analyzed during the current study are available from the corresponding author on reasonable request.

\section{Authors' contributions}

CE participated in the study design performed the plasma preparation and analyses, contributed to the statistical analysis, data interpretation and discussion of results and wrote the manuscript. FB collected the blood samples and helped in plasma preparation and analyzing the samples. AM conceived the study and participated in project planning and coordination. NB participated in project planning, performing the statistical analysis, data interpretation, discussing the results and writing the manuscript. All authors read and approved the final manuscript.

\section{Ethics approval and consent to participate}

Ethical approval has been given by the State Agency for Nature, Environment and Consumer Protection of North Rhine-Westphalia (LANUV NRW, reference number: 84-02.05.20.13.090).

\section{Consent for publication}

Not applicable.

\section{Competing interests}

FB is employed by Bayer Animal Health $\mathrm{GmbH}$, which has no financial interest with the subject matter, the materials used or the performance of the study. CE, AM and NB declare that they have no competing interests in the study.

\section{Publisher's Note}

Springer Nature remains neutral with regard to jurisdictional claims in published maps and institutional affiliations.

\section{Author details}

'Department of Veterinary Clinical Sciences, Clinical Pathophysiology and Clinical Pathology, Justus-Liebig University Giessen, Frankfurterstraße 126, 35392 Giessen, Germany. ${ }^{2}$ Bayer Animal Health GmbH, Alfred-Nobel-Straße 50, 40789 Monheim am Rhein, Germany.

Received: 22 December 2016 Accepted: 16 November 2017

Published online: 29 November 2017

\section{References}

1. Mallett SV, Cox DJ. Thrombelastography. Br J Anaesth. 1992;69:307-13.

2. Bauer N, Eralp O, Moritz A. Effect of hemolysis on canine kaolin-activated thromboelastography values and ADVIA 2120 platelet activation indices. Vet Clin Pathol. 2010;39:180-9.

3. Wiinberg $B$, Jensen $A L$, Rojkjaer $R$, Johansson $P$, Kjelgaard-Hansen $M$ Kristensen AT. Validation of human recombinant tissue factor-activated thromboelastography on citrated whole blood from clinically healthy dogs. Vet Clin Pathol. 2005;34:389-93.

4. Rivard GE, Brummel-Ziedins KE, Mann KG, Fan L, Hofer A, Cohen E. Evaluation of the profile of thrombin generation during the process of whole blood clotting as assessed by thrombelastography. J Thromb Haemost. 2005;3:2039-43. doi:10.1111/j.1538-7836.2005.01513.x.

5. Pommerening MJ, Goodman MD, Farley DL, Cardenas JC, Podbielski J, Matijevic N, et al. Early diagnosis of clinically significant hyperfibrinolysis using thrombelastography velocity curves. J Am Coll Surg. 2014;219:115766. doi:10.1016/j.jamcollsurg.2014.07.943.

6. Gonzalez E, Kashuk JL, Moore EE, Silliman CC. Differentiation of enzymatic from platelet hypercoagulability using the novel thrombelastography parameter delta (delta). J Surg Res. 2010;163:96-101. doi:10.1016/j.jss.2010.03.058.

7. Ellis TC, Nielsen VG, Marques MB, Kirklin JK. Thrombelastographic measures of clot propagation: a comparison of alpha with the maximum rate of thrombus generation [abstract]. Blood Coagul Fibrinolysis. 2007;18:45-8. doi: 10.1097/MBC.0b013e3280111a8e

8. McMichael MA, Smith SA. Viscoelastic coagulation testing: technology, applications, and limitations. Vet Clin Pathol. 2011;40:140-53. doi:10.1111/j. 1939-165X.2011.00302.x.

9. Haemonetics Corporation. TEG ${ }^{\circledR} 5000$ System - User Manual - 2011.

10. Donahue SM, Otto CM. Thromboelastography: a tool for measuring hypercoagulability, hypocoagulability, and fibrinolysis. J Vet Emerg Crit Care (San Antonio). 2005;15:9-16. doi:10.1111/j.1476-4431.2005.04025.x.

11. Wiinberg B, Kristensen AT. Thromboelastography in veterinary medicine. Semin Thromb Hemost. 2010:36:747-56. doi:10.1055/s-0030-1265291.

12. Kashuk JL, Moore EE, Sabel A, Barnett C, Haenel J, Le T, et al. Rapid thrombelastography ( $r$-TEG) identifies hypercoagulability and predicts thromboembolic events in surgical patients. Surgery. 2009;146:764-772; discussion 772-4. doi:10.1016/j.surg.2009.06.054.

13. Flatland B, Koenigshof AM, Rozanski E, Goggs R, Wiinberg B. Systematic evaluation of evidence on veterinary viscoelastic testing part 2: sample acquisition and handling. J Vet Emerg Crit Care (San Antonio). 2014;24:30-6. doi:10.1111/vec.12142.

14. Flint SK, Wood D, Abrams-Ogg AC, Kruth SA, Bersenas A. Comparison of citrated native and kaolin-activated samples for thrombelastographic analysis in healthy dogs. Vet Clin Pathol. 2012;41:249-55. doi:10.1111/j.1939165X.2012.00431.x.

15. White H, Zollinger C, Jones M, Bird R. Can Thromboelastography performed on kaolin-activated citrated samples from critically ill patients provide stable and consistent parameters? Int J Lab Hematol. 2010;32:167-73. doi:10.1111/j. 1751-553X.2009.01152.x.

16. Zambruni A, Thalheimer U, Leandro G, Perry D, Burroughs AK Thromboelastography with citrated blood: comparability with native blood, 
stability of citrate storage and effect of repeated sampling. Blood Coagul Fibrinolysis. 2004;15:103-7.

17. Leclere M, Lavoie J, Dunn M, Bedard C. Evaluation of a modified thrombelastography assay initiated with recombinant human tissue factor in clinically healthy horses. Vet Clin Pathol. 2009;38:462-6. doi:10.1111/j. 1939-165X.2009.00157.x.

18. Garreis K, Palmer S, Sommerey C, Klein R, Müller E. Investigation of sample stability of kaolin activated citrated whole blood and establishment of reference values for thromboelastography in healthy adult horses [abstract]. Tierarztl Prax Ausg K Kleintiere Heimtiere. 2015;2:A11.

19. Banerjee A, Blois SL, Wood D. Comparing citrated native, kaolin-activated, and tissue factor-activated samples and determining intraindividual variability for feline thromboelastography. J Vet Diagn Investig. 2011;23: 1109-13. doi:10.1177/1040638711425595.

20. Hall DJ, Rush JE, deLaforcade AM, Shaw SP. Kaolin-activated thromboelastography in echocardiographically normal cats. Am J Vet Res. 2012;73:775-8. doi:10.2460/ajvr.73.6.775.

21. Marschner CB, Bjørnvad CR, Kristensen AT, Wiinberg B. Thromboelastography results on citrated whole blood from clinically healthy cats depend on modes of activation. Acta Vet Scand. 2010;52:38. doi:10.1186/1751-0147-52-38.

22. Moldal ER, Kirpensteijn J, Kristensen AT, Haga HA, Nødtvedt A, Eriksen T. Evaluation of inflammatory and hemostatic surgical stress responses in male cats after castration under general anesthesia with or without local anesthesia. Am J Vet Res. 2012;73:1824-31. doi:10.2460/ajvr.73.11.1824.

23. Cöl R, Monthomery A, lazbik MC, Defelice A, Saavedra PV, Couto CG. Wholeblood thrombelastography using calcium chloride activation in healthy cats. Turk J Vet Anim Sci. 2013;37:68-75.

24. Alwood AJ, Downend AB, Slensky KA, Fox JA, Simpson SA, Donahue SM, et al. Resident forum abstracts: evaluation of thrombelastography (TEG) in normal cats. J Vet Emerg Crit Care (San Antonio). 2004;14:S1-S17. doi:10. 1111/j.1476-4431.2004.04035.x.

25. Bauer N, Eralp Inan O, Moritz A. Reference intervals and method optimization for variables reflecting hypocoagulatory and hypercoagulatory states in dogs using the STA compact automated analyzer. J Vet Diagn Investig. 2009;21(6):803-14.

26. Moritz A, Fickenscher Y, Meyer K, Failing K, Weiss DJ. Canine and feline hematology reference values for the ADVIA 120 hematology system. Vet Clin Pathol. 2004;33:32-8. doi:10.1111/j.1939-165X.2004.tb00347.x.

27. Tasker S, Cripps PJ, Mackin AJ. Estimation of platelet counts on feline blood smears. Vet Clin Pathol. 1999;28:42-5.

28. Geffré A, Concordet D, Braun J-P, Trumel C. Reference value advisor: a new freeware set of macroinstructions to calculate reference intervals with Microsoft excel. Vet Clin Pathol. 2011;40(1):107-12. doi:10.1111/j.1939-165X. 2011.00287.x.

29. Friedrichs KR, Harr K, Freeman KP, Szladovits B, Walton RM, Barnhart KF, Blanco-Chavez J. ASVCP reference interval guidelines: determination of de novo reference intervals in veterinary species and other related topics. Vet Clin Pathol. 2012;41:441-53. doi:10.1111/vcp.12006.

\section{Submit your next manuscript to BioMed Central and we will help you at every step:}

- We accept pre-submission inquiries

- Our selector tool helps you to find the most relevant journal

- We provide round the clock customer support

- Convenient online submission

- Thorough peer review

- Inclusion in PubMed and all major indexing services

- Maximum visibility for your research

Submit your manuscript at www.biomedcentral.com/submit 